\title{
SOME ASPECTS OF RADIO PULSAR EVOLUTION
}

\author{
V. S. Beskin, A. V. Gurevich and YA. N. Istomin \\ P. N. Lebedev Physical Institute
}

It is shown that pulsar statistics are in good agreement with the theory of current (plasma) energy losses of a neutron star. Up to now two theories of pulsar braking have been considered. The first well known model is connected with the radiation of vacuum magneto-dipole waves (Ostriker and Gunn 1969). However, according to the electrodynamical theory of pulsars, the presence of a rather dense plasma in the magnetosphere of a neutron star leads inevitably to a complete suppression of magneto-dipole losses-the dynamics of the stellar slow down is determined by the longitudinal current only (Beskin, Gurevich, and Istomin 1983). In the table below we present the main differences between the predictions of these two theories:

Table 1 Predictions of the theories

\begin{tabular}{|c|c|c|}
\hline & vacirum inodel & current model \\
\hline energy losses & $W \simeq \frac{B_{0}^{2} \Omega^{4} R^{6}}{c^{3}} \sin ^{2} \chi$ & $W \simeq \frac{B_{0}^{2} \Omega^{4} R^{6}}{c^{3}} i_{0} \cos \chi$ \\
\hline invariant of motion & $I=\Omega \cos \chi$ & $I=\Omega \sin \chi$ \\
\hline $\begin{array}{l}\text { evolution of the } \\
\text { inclination angle } \chi\end{array}$ & $d x / d t<0$ & $d \chi / d t>0$ \\
\hline braking index & $n_{\mathrm{b}}=3+2 \ldots$ & $=1.93+1.5 \tan ^{2} \chi$ \\
\hline magnetic field & $B_{12} \cong\left(P \dot{P}_{15}\right)^{\prime}$ & $B_{12} \cong P^{i / 10} \dot{P}_{15}^{-1 / 2}$ \\
\hline determination & & $B_{12} \cong p^{15 / 8}$ \\
\hline
\end{tabular}

We see that these theories predict an opposite evolution of the inclination angle $\chi$. It is necessary to stress that the current model gives unusual values of the magnetic field. Finally, the current theory is different for pulsars with $Q>1$ and $Q<1$.

Unfortunately, we have no precise information now about the actual evolution of radio pulsars. One reason is connected with the fact that the kinetic equation

$$
\frac{\partial}{\partial P}\left(N \frac{d P}{d t}\right)+\frac{\partial}{\partial \chi}\left(N \frac{d \chi}{d t}\right)+\frac{\partial}{\partial B}\left(N \frac{d B}{d t}\right)=B-D
$$

describing the pulsar distribution function $N(P, X, B)$ not only has several unknown parameters (such as the magnetic field time decay $\tau_{B}$ ) but also unknown functions (birth function $B$ and death function $D$ ). So it is not surprising that even for the vacuum-losses theory there are several points of view on the initial pulsar periods $P_{0}$ and the time scale of the magnetic field evolution. Similarly, in the case of the theory of plasma losses, we find that its predictions are also in good agreement with observational data (Beskin, Gurevich, and Istomin 1984). Here we present some additional arguments in favor of this theory:

1. Now it is rather difficult to explain the heating of the less massive companion in the eclipsing binary system on the basis of vacuum magneto-dipole radiation, because it is necessary to have a flux of particles and/or $\gamma$-rays containing almost all of the braking energy. The theory of current losses provides us with just such a particle flux.

2. Irrespective of the slowing down mechanism, pulsars which have appreciably changed their period during the time of their evolution must appreciably change their inclination angle $\chi$. One such 
pulsar is PSR $0531+21$ (the Crab Nebula pulsar) whose dynamical age $\tau_{\mathrm{D}}=2400 \mathrm{yr}$ considerably exceeds its real age of $936 \mathrm{yr}$. This means that during its lifetime the rotational frequency $\Omega$ has lowered significantly. Therefore, the inclination angle of this pulsar is at present close to $90^{\circ}$, which is undoubtedly evidence that this angle has actually increased with time.

3. Recently Lyne and Manchester (1988), Rankin (1990) and Malov (1990) have given complete catalogs of inclination angles $\chi$ which make it possible to carry out detailed comparisons of theoretical results with the observations. Figures $1 \mathrm{a}$ and $1 \mathrm{~b}$ give the distribution of pulsars over angle $\chi$ separately for pulsars with $Q>1$ and $Q<1$. The curves correspond to the theory derived from the kinetic equation eq.(1). We see that there is no difference between "young" ( $\tau_{\mathrm{D}}<2 \times 10^{6} \mathrm{yr}$ ), and "old" $\left(\tau_{\mathrm{D}}>2 \times 10^{6} \mathrm{yr}\right)$ pulsars with $Q<1$. The pulsars with $Q>1$, shown in figure 1c, really must, however, have a deficit for $\chi \simeq 90^{\circ}$.

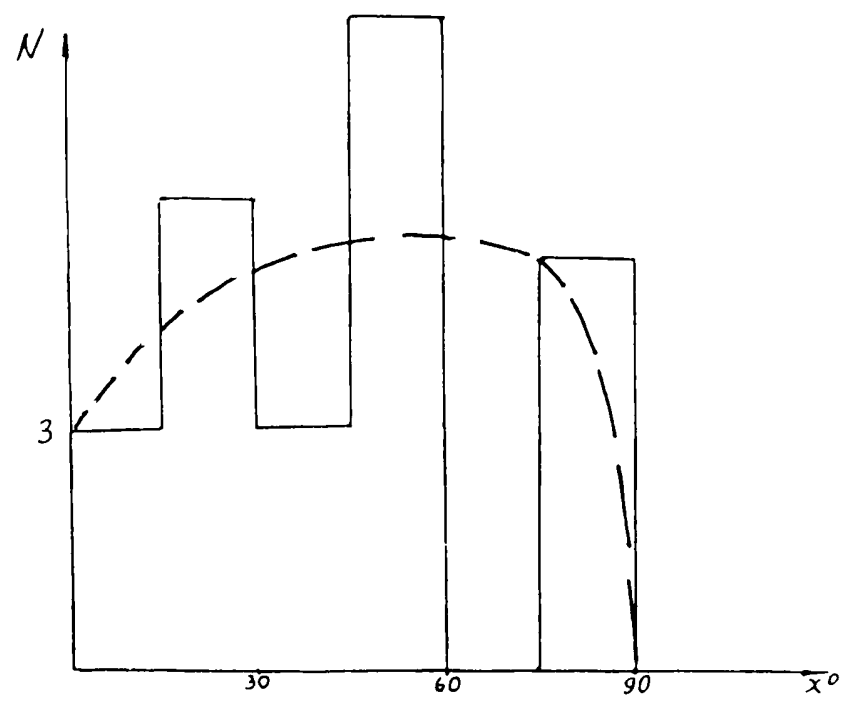

Figure 1a Distribution of pulsars over the angle $\chi$ with $Q<1$ and $\tau_{\mathrm{D}}<2 \times 10^{6}$ years.



Figure 1c Distribution of pulsars over the angle $\chi$ with $Q>1$

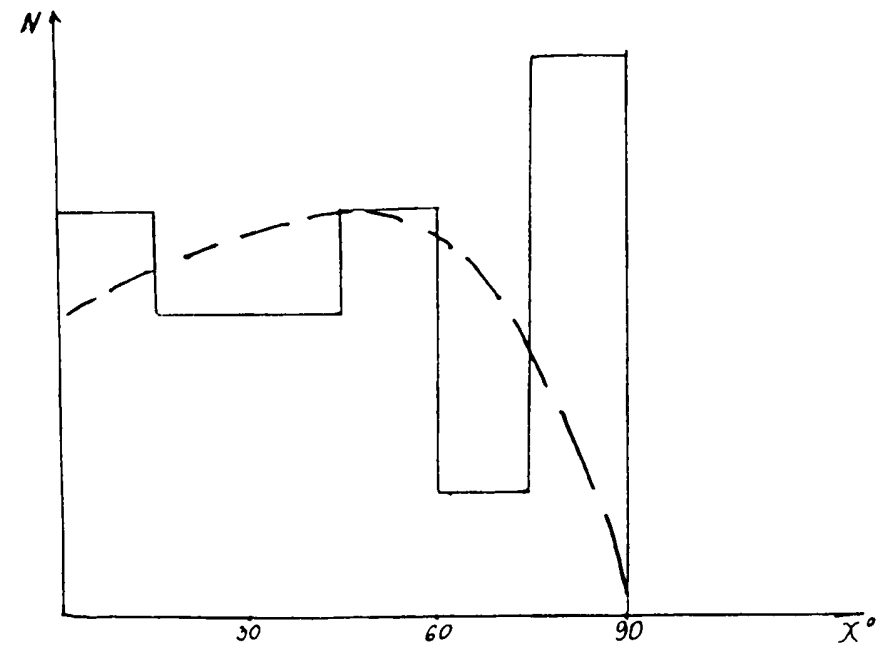

Figure 1b Distribution of pulsars over the angle $\chi$ with $Q<1$ and $\tau_{\mathrm{D}}>2 \times 10^{6}$ years.

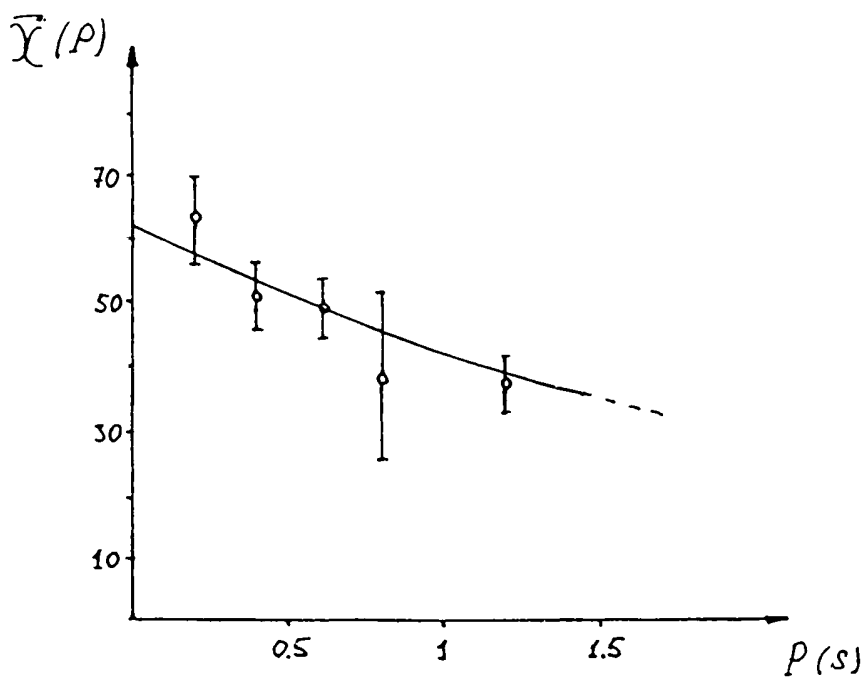

Figure 2 Mean values $\bar{\chi}(P)$.

Accordingly, the mean value $\bar{\chi}(P)$, as is shown in figure 2 , decreases as $P$ increases, in spite of the fact that for each pulsar, the angle $\chi$ increases with time. The reason for this is that pulsars having $Q<1$ and large periods $P$ cannot have angles $\chi$ close to $90^{\circ}$. The region $Q<1$ on the $P$ vs. $\sin \chi$ diagram is shown in figure 3. One can see that the generation of particles near the pulsar surface with a large enough period $P$ is only possible when $\chi$ is small. Thus, the observed decrease of the mean angle $\bar{\chi}(P)$ cannot be regarded as an argument in favor of the decrease of $\chi$ with time. This is 


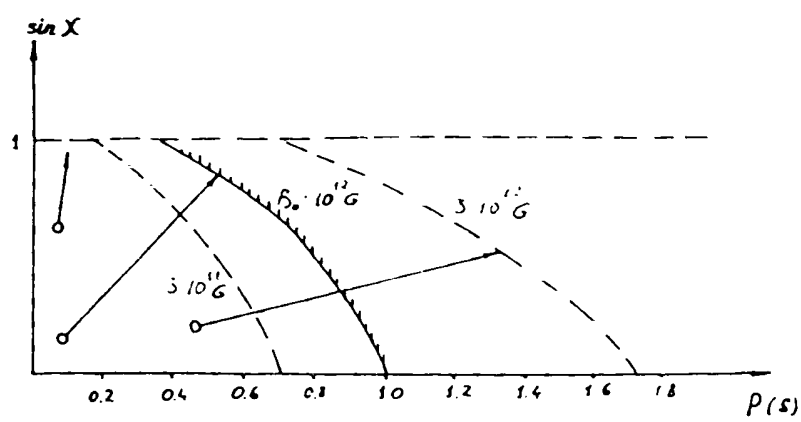

Figure 3

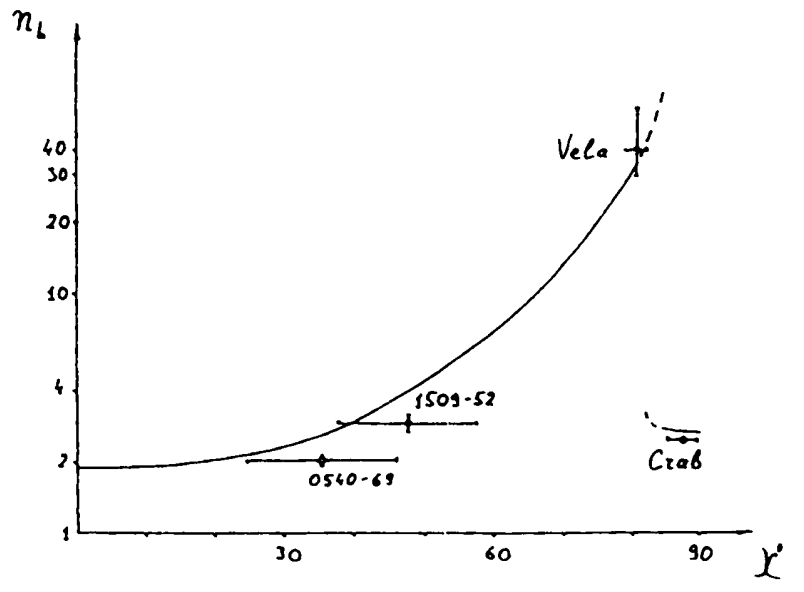

Figure 4

rather an indication that the observed radio emission is indeed associated with particle generation near the stellar surface.

4. According to plasma-losses theory, the braking index

$$
\begin{array}{ll}
n_{\mathrm{b}}=1.93+1.5 \tan ^{2} \chi & \chi \neq 90^{\circ} \\
n_{\mathrm{b}} \approx 2.6 & \chi \approx 90^{\circ}
\end{array}
$$

can be less than three as distinguished from the magneto dipole mechanism where $n_{b} \geq 3$. As is shown in figure 4, we have close agreement between the current theory and the observations. On the other hand, it is difficult to explain values $2<n_{b}<3$ on the basis of vacuum theory. 